\title{
Medidas preventivas de pneumonia associada à ventilação mecânica
}

\author{
Preventive mersures of pneumonia associated with mechanical ventilation \\ Medidas preventivas de neumonía associada a ventilación mecânica
}

Gabriel da Costa Sousa

ORCID: https://orcid.org/0000-0002-2642-0885 Centro Universitário Uninovafapi, Brasil E-mail: gcostasousa05@ hotmail.com

Kelly Evenlly da Silva Santos

ORCID: https://orcid.org/0000-0002-6217-5143 Centro Universitário Uninovafapi, Brasil E-mail: kelly.evenlly@hotmail.com

Lana Borges da Silva

ORCID: https://orcid.org/0000-0002-6557-5433 Centro Universitário Uninovafapi, Brasil E-mail: lanaborges12.08@gmail.com Jadilson Rodrigues Mendes

ORCID: https://orcid.org/0000-0001-6164-4772 Centro Universitário Uninovafapi, Brasil

E-mail: jadilson.mendes@uninovafapi.edu.br Magda Rogéria Pereira Viana

ORCID: https://orcid.org/0000-0003-3293-7095 Centro Universitário Uninovafapi, Brasil E-mail: magdarogeria@ @otmail.com

Sarai de Brito Cardoso

ORCID: https://orcid.org/0000-0002-8941-1038 Centro Universitário Uninovafapi, Brasil E-mail: sarai.cardoso@uninovafapi.edu.br

\begin{abstract}
Resumo
Objetivou-se analisar as medidas de prevenção à pneumonia associada à ventilação mecânica evidenciadas na literatura científica. Trata-se de um estudo de revisão integrativa. Para a elaboração da questão de pesquisa da revisãointegrativa, utilizou-se a estratégia PICO. A presente pesquisa foi feita mediante buscaonline nas bases de dados da Biblioteca Virtual em Saúde (BVS) no período de 2015 a 06/2020.Após a leitura criteriosa dos artigos, foram avaliados 19 artigos que atenderam aos critérios de inclusão previamente estabelecidos. Dessa forma foram definidas as seguintes categorias após análise por similaridade dos conteúdos: Categoria I- Ações de prevenção para oenfrentamento da PAV e Categoria II- Estratégia de promoção para enfrentamento da PAV. O estudo mostra que a implementação do bundle, evidenciou ser uma importante ferramenta que deve ser incorporada diariamente na assistência,reduzindo o risco de pneumonia associada à ventilação mecânica que se torna uma complicação decorrente ao paciente hospitalizado.
\end{abstract}

Palavras-chave: Pneumonia; Prevenção; Ventilação mecânica.

\begin{abstract}
The objective was to analyze the preventive measures for mechanical ventilation associated pneumonia, as evidenced in the scientific literature. This is an integrative review study. For the elaboration of the research question, the PICO strategy was used. This research was done through online search in the databases of the Biblioteca Virtual em Saude (BVS) in the period from 2015 to $06 / 2020$. After carefully reading the articles, 19 articles that met the inclusion criteria previously established were evaluated. This way, the following categories were defined after analysis for similarity of content: Category I- Preventive actions to face the PAV and Category II- Promotion of strategies to face the PAV. The study shows that the implementation of the bundle, proved to be an important tool that should be incorporated daily in care, reducing the risk of mechanical ventilation associated pneumonia, which becomes a resulted complication in hospitalized patients.
\end{abstract}

Keywords: Pneumonia; Prevention; Mechanical ventilation.

\section{Resumen}

El objetivo fue analizar las medidas preventivas de la neumonía asociada a la ventilación mecánica, como se evidencia en la literatura científica. Se trata de un estudio de revisión integradora. Para la elaboración de la cuestión de investigación de la revisión integradora, se utilizó la estrategia PICO. Esta investigación se realizó mediante búsqueda en línea en las bases de datos de la Biblioteca Virtual en Salud (BVS) en el período de 2015 a 06/2020. Después de la 
lectura minuciosa de los artículos, fueron evaluados 19 artículos que cumplieron con los criterios de inclusión previamente establecidos. Así, se han definido as siguientes categorías después de análisis de similitud de contenido: Categoría I- Acciones de prevención para enfrentamiento de la PAV y Categoría II- Estrategia de promoción para enfrentamiento de la PAV. El estudio muestra que la implementación del bundle demostró ser una herramienta importante que debe incorporarse diariamente en la asistencia, reduciendo el riesgo de neumonía asociada a la ventilación mecánica, que se convierte en una complicación decurrente al paciente hospitalizado.

Palabras clave: Neumonía; Prevención; Ventilación mecánica.

\section{Introdução}

Pereira e Araújo (2017) definem IRAS como infecções adquiridas durante a prestação dos cuidados de saúde e representam um dos mais importantes problemasde saúde pública no mundo, gerando consideráveis aumento nos custos, tempo de internação, morbidade e mortalidade.

A Agência Nacional de Vigilância Sanitária (2015), no Brasil as primeiras Comissões de Controle de Infecção Hospitalar (CCIH) surgiram na década de 60, trazendo grandes mudanças na assistência aos enfermos. Com as constantes evoluções ocorridas nas $\mathrm{CCIH}$, bem como os movimentos dos profissionais da saúde, a concepção de infecção hospitalar também mudou, passando a ser considerada atualmente como Infecções Relacionadas à Assistência à Saúde (IRAS) e essa temática é considerada um problema de saúde pública no mundo pelo alto custo gerado.

Segundo Rau e Santos (2015) uma das IRAS é a pneumonia, doença inflamatória que acomete o parênquima pulmonar, bronquíolos e alvéolos fazendo com que as trocas gasosas sejam prejudicadas, podendo ser dividida em dois tipos: pneumonia comunitária (PAC), adquirida por qualquer indivíduo na comunidade, assim como pneumonia hospitalar (PAH), sendo uma das principais causas de morte em pacientes hospitalizados, principalmente os que se beneficiam de tratamento emUTI e que precisam de ventilação mecânica (VM), haja vista que a VM frequentementeé apontada como um dos principais fatores de risco para o desenvolvimento de PAH.

É correto afirmar que grande parte das infecções hospitalares podem ser evitadas com a adoção de medidas preventivas que estejam apoiadas emconhecimentos técnicos e científicos adequados, pois a pneumonia é responsável por $15 \%$ das IRAS e aproximadamente $25 \%$ de todas infecções adquiridas em Unidadesde Terapia Intensiva, ela é a segunda infecção mais comum em UTI. Observando que sua relevância se dá pelo aumento de procedimentos invasivos, pelo uso indiscriminado de antimicrobianos e pelo aumento de internação hospitalar (Maran et al., 2019).

Sabe-se que a maior parte dos pacientes graves internados em uma UTI precisa de VM para manter as necessidades fisiológicas de oxigenação em casos deinsuficiência respiratória (Santos et al., 2018).

Segundo Dutra et al (2019) Pneumonia Associada à ventilação mecânica (PAV) é uma IRAS que se inicia de 48 a 72 horas após a intubação endotraqueal e o estabelecimento da ventilação mecânica invasiva, e que tem, como agente causador, um microrganismo que não estava presente ou incubado e cuja ocorrência, geralmente, decorre da aspiração de secreções das vias áreas superiores, do refluxo gastrintestinal e da inoculação de material exógeno contaminado.

Uma das principais patogenias da PAV é a secreção carregada de micróbios, que vai se espalhando das vias aéreas superiores para as partes baixas dos pulmõespor meio de microaspiração ou via endotraqueal. Em estudos anteriores, mediações foram propostas a fim de reduzir a incidência de PAV, incluindo elevação da cabeceirada cama, teste diário de desmame do ventilador, higiene das mãos com programa deeducação do pessoal e cuidados bucais com gluconato de clorexidina. Vale destacar que os patógenos da PAV são de forma constante geneticamente diferentes das cepas isoladas da cavidade oral, o que indica a importância da higienização bucal naprevenção da PAV (Su et al., 2017).

Medidas de precaução e cuidados para evitar PAV têm sido um desafio para os serviços de saúde, em especial na UTI. O foco é pensar em estratégias de prevenção, controle desta infecção, e, por fim, redução de suas taxas de incidência com a aplicação de boas práticas assistenciais que devem ser aplicadas no dia a dia, como por exemplo o treinamento constante das equipes, visto 
que medidas preventivas têm sido eficazes para a melhora na qualidade da assistência, quando executadas de forma contínua e coletiva por todos os profissionais de saúde (Alecrim et al., 2019). A partir dessas considerações, esse estudo teve como objetivo identificar e analisar as medidas de prevenção à pneumonia associada à ventilação mecânica evidenciadas na literatura científica.

\section{Métodos}

Trata-se de um estudo de revisão integrativa da literatura, que de acordo com Freire, Sawad, de França, da Costa, \& Oliveira (2014), tem sido utilizada como recurso metodológico, a qual faz uso de estratégia sistematizada para agrupar, bem como produzir resultados de estudos sobre um determinado tema, com o objetivo de aprofundar e reforçar o conhecimento científico das áreas em questão, assim como subsidiar a tomada de decisões dos profissionais.

Para a construção de uma revisão integrativa, é necessário seguir seis etapas distintas: a identificação do tema e seleção da hipótese ou questão de pesquisa; estabelecimento de critérios para inclusão e exclusão de estudos/amostragem ou busca na literatura; definição das informações a serem extraídas dos estudos selecionados/categorização dos estudos; avaliação dos estudos incluídos; interpretação dos resultados; e apresentação da revisão/síntese do conhecimento (Ercole; Melo; \& Alcoforado, 2014).

Para a elaboração da questão de pesquisa da revisão integrativa, utilizou-se aestratégia PICO (acrônimo para patient, intervention, comparison, outcomes). $\mathrm{O}$ uso dessa estratégia para formular a questão de pesquisa na condução de métodos de revisão possibilita a identificação de palavras-chave, as quais auxiliam na localização de estudos primários relevantes nas bases de dados (Melnyk, Fineout-overholt, 2011).

Assim, a questão de pesquisa delimitada foi: "Quais medidas devem ser adotadas por profissionais da saúde para prevenção de Pneumonia Associada à Ventilação Mecânica (PAV)?". Nela, o primeiro elemento da estratégia (P) consiste no profissional de saúde; o segundo (I), as medidas adotadas; e o quarto elemento (O) prevenção de PAV. Ressalta-se que, dependendo do método de revisão, não se emprega todos os elementos da estratégia PICO. Nesta revisão integrativa, o terceiro elemento, ou seja, a comparação, não foi utilizada.

A coleta de dados foi elaborada nos meses de setembro e outubro de 2020, através da busca de dados na Biblioteca Virtual em Saúde (BVS), com isso utilizou-se as bases de dados da Literatura Latino-Americano e do Caribe em Ciências da Saúde (LILACS), Base de Dados da Enfermagem (BDENF), Biblioteca Eletrônica Científica Online (SCIELO), MEDLINE e Web of Science. Aplicando os seguintes descritores/Mesh: Pneumonia/pneumonia, Prevenção/prevention, Ventilação Mecânica/mechanical ventilation. A busca dos artigos ocorreu com a associação dos descritores ao operador boleano AND. E todo o processo de avaliação e seleção dosartigos são demonstrados da Figura 1.

Foram utilizados para desenvolvimento da pesquisa os seguintes critérios de inclusão, artigos científicos disponíveis na íntegra no período de 01/2015 a 10/2020 que estejam escritos nos idiomas português, espanhol ou inglês. Quanto aos critérios de exclusão, foram levados em consideração artigos do tipo revisão bibliográfica, quenão atendam ao objetivo da pesquisa e que estejam duplicados.

Na Prática Baseada em Evidência existe uma classificação de evidênciacientífica caracterizadas de forma hierárquica, dependendo da abordagem metodológica adotada, os estudos são classificados em níveis de evidência de I a VI, e os quais são apresentados a seguir, Quadro 1.

Para o registro e organização das informações dos artigos selecionados para a revisão, foi elaborado um instrumento de coleta de dados, contendo questões relacionadas a título do artigo, autores, ano de publicação, nível de evidência científicae resultados. Estes estudos foram codificados pela letra A (Artigo) seguidos por números (de 1 a 19), para melhor organização, apresentação e análise dos dados. 
Após a coleta, assim como a leitura dos artigos, os mesmos foram organizadosem quadro e realizadas a criação de categorias que respondem aos objetivos do estudo, realizando a discussão de acordo com os resultados dos artigos trabalhados.

Figura 1: Fluxograma da busca de artigos na base de dados para inclusão na revisão. Teresina, PI, no período de 2015 a 2020.

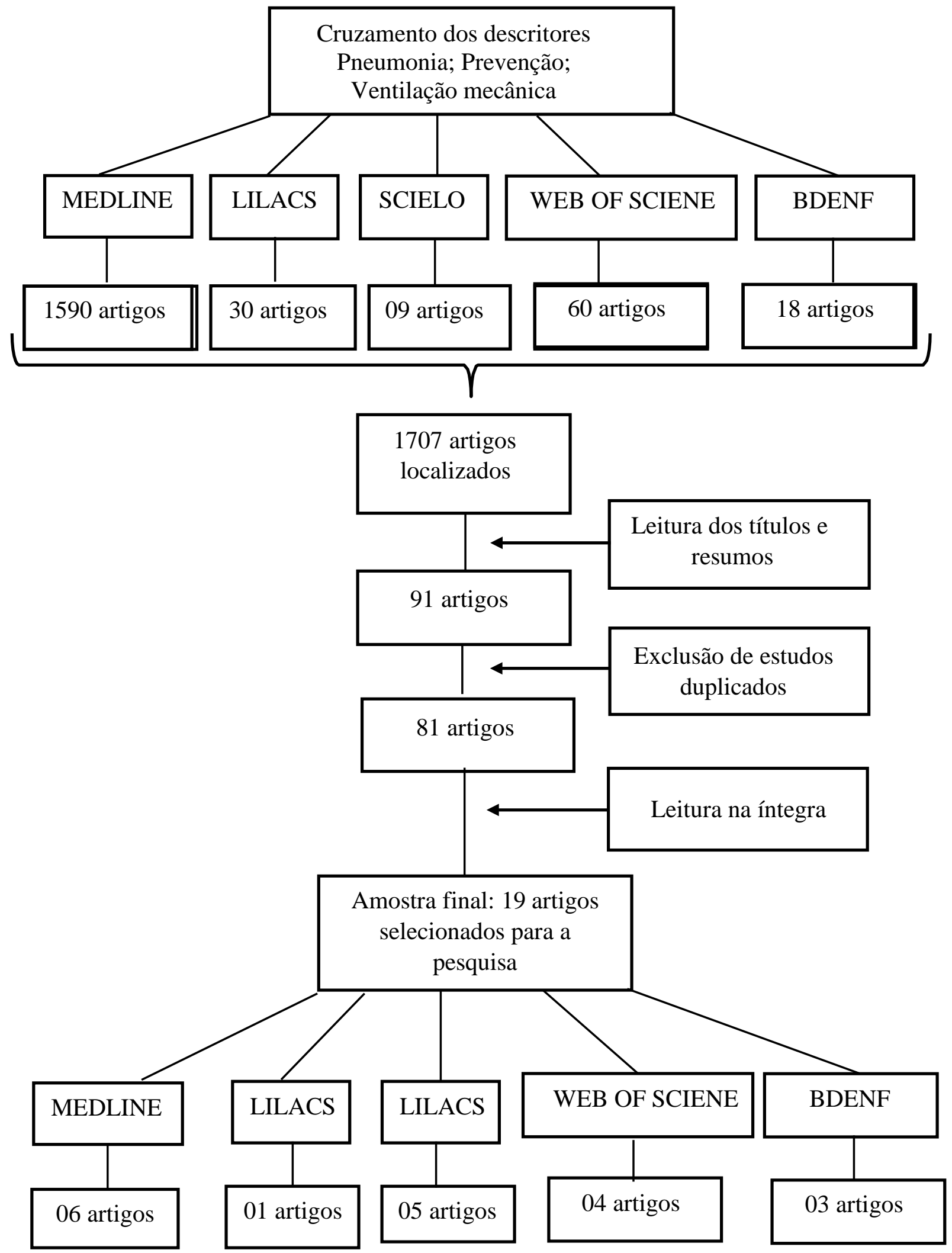

Fonte: Autores. 
Quadro 1: Classificação de evidência científica segundo a base de dados SCIELO.

\begin{tabular}{|c|c|}
\hline Nível I & $\begin{array}{c}\text { Evidências resultantes de meta-análise de múltiplos estudos clínicos } \\
\text { controlados e randomizados. }\end{array}$ \\
\hline Nível II & $\begin{array}{c}\text { Evidências obtidas em estudos individuais com delineamento } \\
\text { experimental. }\end{array}$ \\
\hline Nível III & Evidências de estudos quase-experimentais. \\
\hline Nível IV & $\begin{array}{c}\text { Evidências de estudos descritivos (não experimentais ou com } \\
\text { abordagem qualitativa). }\end{array}$ \\
\hline Nível V & Evidências provenientes de relatos de caso ou de experiência. \\
\hline Nível VI & Evidências baseadas em opiniões de especialistas. \\
\hline
\end{tabular}

Fonte: Botelho, de Almeida Cunha, e Macedo (2011).

\section{Resultados}

Nesta revisão integrativa foram avaliados 19 artigos que atenderam aos critérios de inclusão previamente estabelecidos. A seguir os resultados serão apresentados através de um quadro se expõe, por meio dos artigos localizados, os focos dados às produções científicas acerca da temática estudada.

Quadro 2: Distribuição das produções científicas segundo o título, autor, ano de publicação, nível de evidência e principal resultado. Teresina, 2020.

\begin{tabular}{|c|c|c|c|c|}
\hline & TITULO & AUTOR/ANO & $\begin{array}{l}\text { NIVEIS DE } \\
\text { EVIDÊNCIA }\end{array}$ & RESULTADOS \\
\hline A-1 & $\begin{array}{c}\text { Adesão ao bundle de prevenção } \\
\text { depneumonia associada à } \\
\text { ventilação mecânica }\end{array}$ & de Barros, 2019. & IV & $\begin{array}{l}\text { O estudou mostrou quea frequência de } \\
\text { adesão ao bundle pelos profissionais teve } \\
\text { significância para três dos seis itens } \\
\text { propostos,sendo eles a posição dacabeceira } \\
\text { entre } 30 \text { a } 45^{\circ} \text {,cuidados com aspiração das } \\
\text { secreções e a fisioterapia } \\
\text { respiratória e motora. }\end{array}$ \\
\hline A-2 & $\begin{array}{c}\text { Impact of oralhygiene in } \\
\text { patients undergoing mechanical } \\
\text { ventilation in the COVID- } 19 \\
\text { pandemic }\end{array}$ & $\begin{array}{l}\text { Silva et al., } \\
2020 .\end{array}$ & IV & $\begin{array}{l}\text { O estudo demostrou a inclusão da } \\
\text { odontologia na assistência de pacientes } \\
\text { internados na UTI de um hospitalpúblico da } \\
\text { cidade deBelo Horizonte, MG. } 100 \% \text { dos } \\
\text { profissionais envolvidos na assistência a } \\
\text { esses pacientes foram favoráveis e } 62 \% \\
\text { reconheceram a contribuição docirurgião- } \\
\text { dentista emconjunto com a equipe de } \\
\text { enfermagem. }\end{array}$ \\
\hline A-3 & $\begin{array}{l}\text { Education to prevent ventilator- } \\
\text { associated pneumonia in } \\
\text { intensive careunit }\end{array}$ & $\begin{array}{c}\text { Branco et al., } \\
2020 .\end{array}$ & III & $\begin{array}{l}\text { A adesão da equipe de enfermagem aos } \\
\text { elementos que compõem o bundle de } \\
\text { prevenção onde houve diminuição da } \\
\text { densidade da incidênciade infecção para os } \\
\text { meses após a aplicação das medidas } \\
\text { preventivas, bem como após o treinamento. } \\
\text { Antes do treinamento, higiene bucal e } \\
\text { escovação dentária, apresentaram baixas } \\
\text { taxas de adesão de } 89,5 \% \text { e } 80,8 \% \text {, } \\
\text { respectivamente. }\end{array}$ \\
\hline
\end{tabular}




\begin{tabular}{|c|c|c|c|c|}
\hline A-4 & $\begin{array}{l}\text { Good practices forpreventing } \\
\text { ventilator- associated } \\
\text { pneumonia in the emergency } \\
\text { department }\end{array}$ & Frota et al., 2019. & IV & $\begin{array}{l}\text { Este estudo avaliou a conformidade do } \\
\text { conjunto de boas práticas para a prevenção } \\
\text { PAV. As cinco medidas de prevenção } \\
\text { de PAV: aelevação da cabeceira, higiene } \\
\text { oral, interrupçãodiária da sedação,profilaxia } \\
\text { de TVP e aprofilaxia de úlcerapeptídica, } \\
\text { apenas duas apresentaram conformidade } \\
\text { maior que } 50 \% \text {, no caso a profilaxia de TVP } \\
\text { e de úlcera péptica. Noentanto os impactos } \\
\text { negativos para esse resultado, ainsuficiência } \\
\text { de recursos materiais e humanos e a } \\
\text { superlotação de pacientes em cuidados } \\
\text { intensivos nos serviços de emergência. }\end{array}$ \\
\hline A-5 & $\begin{array}{l}\text { Pneumonia associada à } \\
\text { ventilação mecânica invasiva: } \\
\text { cuidados deenfermagem }\end{array}$ & Cruz, 2018. & IV & $\begin{array}{l}\text { Após as avaliações dos enfermeiros, pôde- } \\
\text { se observar a qualificação e a boa } \\
\text { percepção de seus conhecimentos acerca } \\
\text { dos cuidados da equipe diante da PAVMI. } \\
\text { Apenas dois novoscasos do total de PAV } \\
\text { em um grupo de } 634 \\
\text { doentes/intubados/dia/ } \\
\text { correspondendo auma taxa de } 0,3 \% \text {. }\end{array}$ \\
\hline A-6 & $\begin{array}{c}\text { Reducing ventilator- associated } \\
\text { pneumonia inneonatal intensive } \\
\text { careunit using "VAP } \\
\text { prevention Bundle": a cohort } \\
\text { study }\end{array}$ & $\begin{array}{c}\text { Azab et al., } \\
2015 .\end{array}$ & II & $\begin{array}{l}\text { Após a implementaçãodo pacote de PAV, } \\
\text { houve uma reduça significantemente do } \\
\text { índice de PAV. Teve uma tendência na } \\
\text { redução de internações, mas a taxa de } \\
\text { mortalidade emcomparação com a faseI e II } \\
\text { não obteve êxodo entre as duas. }\end{array}$ \\
\hline A-7 & $\begin{array}{l}\text { Impact of VAP bundle } \\
\text { adherence among ventilated } \\
\text { critically ill patients and its } \\
\text { effectivenessin adult ICU }\end{array}$ & $\begin{array}{l}\text { Samra, Sherif, } \\
\text { Elokda, } 2017 .\end{array}$ & I & $\begin{array}{l}\text { O desfecho do estudo foianalisar a adesão do } \\
\text { pacote PAV, para isso os pesquisadores } \\
\text { dividiram os pacientes em dois grupos, A e } \\
\text { B, logo após sesubdividiram em com PAV } \\
\text { e sem PAV. Os pacientes do grupo A não } \\
\text { receberam o protocolo do pacote PAV, já o } \\
\text { grupo B receberam. E foiconcluído que a } \\
\text { taxa de mortalidade diminuiu no grupo B em } \\
\text { ambos os subgrupos do que no grupo } \\
\text { A, e o tempo de permanência na UTI foi } \\
\text { reduzido. }\end{array}$ \\
\hline A- 8 & $\begin{array}{l}\text { Adesão às medidas preventivas } \\
\text { versus incidência depneumonia } \\
\text { associada à ventilação mecânica }\end{array}$ & $\begin{array}{c}\text { Lourençone, } \\
\text { Branco, Monteiro, } \\
\text { Fonseca, } \\
\text { Caregnato, } 2019\end{array}$ & IV & $\begin{array}{l}\text { Após a reestruturação do protocolo de } \\
\text { prevenção da PAV, em seis meses de } \\
\text { monitorização, o estudo demonstrou que a } \\
\text { vigilância e o reforçocontínuo da equipe de } \\
\text { enfermagem para a realização das medidas } \\
\text { preventivas, alcançaram índices de adesão } \\
\text { acimados } 77 \% \text {, simultaneamente } \\
\text { diminuição nos de PAV. }\end{array}$ \\
\hline A-9 & $\begin{array}{c}\text { Adesão às medidas de } \\
\text { prevenção para pneumonia } \\
\text { associada àventilação } \\
\text { mecânica }\end{array}$ & $\begin{array}{c}\text { de Almeida, } \\
2015\end{array}$ & IV & $\begin{array}{l}\text { O estudo demonstrou que a execução de } \\
\text { medidas de prevenção baseadas em } \\
\text { evidênciascientíficas pode reduzira PAV de } \\
\text { forma significativa e sustentada, trazendo } \\
\text { segurança na assistência, da mesma foram } \\
\text { que redução de custos, livrando o paciente } \\
\text { de todas as implicações clínicas que uma } \\
\text { infecção do trato respiratório hospitalar. }\end{array}$ \\
\hline
\end{tabular}




\begin{tabular}{|c|c|c|c|c|}
\hline A-10 & $\begin{array}{l}\text { The barriers to the prevention of } \\
\text { ventilator- associated } \\
\text { pneumonia from the perspective } \\
\text { of critical carenurses: A } \\
\text { qualitative descriptive study }\end{array}$ & $\begin{array}{l}\text { Atashi, Yousefi, } \\
\text { Mahjobipoor, \& } \\
\text { Yazdannik, } 2018\end{array}$ & IV & $\begin{array}{l}\text { As barreiras à prevenção } \\
\text { pneumonia associada à ventilação } \\
\text { mecânica seenquadram } \\
\text { categorias principais, a saber, competência } \\
\text { profissional limitada dos enfermeiros, } \\
\text { condições ambientais desfavoráveis e } \\
\text { gerenciamento passivode recursos humanos. }\end{array}$ \\
\hline A-11 & $\begin{array}{l}\text { Nurses' implementati on of } \\
\text { ventilator- associated } \\
\text { pneumonia prevention } \\
\text { guidelines: anobservationalstudy } \\
\text { in Jordan }\end{array}$ & Aloush, 2018 & IV & $\begin{array}{l}\text { A adesão dos enfermeiros foi considerada } \\
\text { insatisfatória. Dos participantes, } 63 \% \\
\text { apresentaram 'adesãoinsuficiente'. }\end{array}$ \\
\hline A-12 & $\begin{array}{l}\text { Analysis of the Preventative } \\
\text { Influence of an Oral Hygiene } \\
\text { Protocol on Ventilator- } \\
\text { AssociatedPneumonia }\end{array}$ & $\begin{array}{l}\text { Scalco et al., } \\
2019\end{array}$ & II & $\begin{array}{l}\text { Este estudo demonstrou que a } \\
\text { implementação deum protocolo de higiene } \\
\text { oral que consiste em limpeza oral com } \\
\text { sucção, uma ação mecânica, aplicada junto } \\
\text { com } 0,12 \% \text { clorexidina, uma ação } \\
\text { química, forneceu um meio eficaz de } \\
\text { prevenção de PAV significativamente em } \\
\text { pacientes sob VM na UTI. }\end{array}$ \\
\hline A-13 & $\begin{array}{l}\text { Impact of a multifaceted } \\
\text { prevention program on } \\
\text { ventilator- associated } \\
\text { pneumonia including selective } \\
\text { oropharyngeal decontamination }\end{array}$ & $\begin{array}{l}\text { Landelle, } \\
\text { et al.,2018 }\end{array}$ & III & $\begin{array}{l}\text { O estudo demonstrouque medidas } \\
\text { preventivas simples, incluindo SOD, } \\
\text { vigilância e feedback, e educação podem } \\
\text { diminuir significativamente as taxas de } \\
\text { PAV, a duraçãoda VM e a mortalidade. } \\
\text { No entanto, o uso de SOD a longo } \\
\text { prazo implica vigilância contínua } \\
\text { do surgimento seleção de resistência } \\
\text { antimicrobiana. }\end{array}$ \\
\hline A-14 & $\begin{array}{l}\text { Protective efect of early low- } \\
\text { dose hydrocortisone } \\
\text { on ventilator-associated } \\
\text { pneumonia in the cancer } \\
\text { patients: a propensity score } \\
\text { analysis }\end{array}$ & $\begin{array}{l}\text { Lagier et al., } \\
2017\end{array}$ & IV & $\begin{array}{l}\text { Encontrado um efeito positivo do } \\
\text { tratamento precoce com hidrocortisona em } \\
\text { baixas doses na prevenção da PAV. } \\
\text { Pacientes com câncer em estado crítico } \\
\text { podemse beneficiar com a administração } \\
\text { de hidrocortisona em baixas doses nos } \\
\text { dias que cercam o inicio da ventilação } \\
\text { mecânica. }\end{array}$ \\
\hline A-15 & $\begin{array}{l}\text { Staff education aimed at reducing } \\
\text { ventilatorasso ciated pneumonia }\end{array}$ & Yilmaz et al., 2016 & IV & $\begin{array}{l}\text { Conscientização do pessoal de seus deveres } \\
\text { devem ser aumentados, o treinamento deve } \\
\text { ser considerado como indispensável para a } \\
\text { prevenção de infecções e prevenir fatores de } \\
\text { risco possíveis devem ser minimizados. } \\
\text { Treinamento reduzido desenvolvimento de } \\
\text { PAV em } 31,7 \% \text {. A mortalidade bruta de } \\
\text { PAV foi de } 69 \% \text { no primeiro período e } 26 \% \\
\text { no segundo. }\end{array}$ \\
\hline A-16 & $\begin{array}{l}\text { Implementing a care bundle } \\
\text { approach reduces } \\
\text { ventilatorassociated pneumonia } \\
\text { and delays ventilatorassociated } \\
\text { tracheobronc hitis in children: } \\
\text { differences according to } \\
\text { endotracheal or tracheostomy } \\
\text { devices }\end{array}$ & $\begin{array}{l}\text { Peña-López et al., } \\
2016\end{array}$ & IV & $\begin{array}{l}\text { Crianças traqueostomizadas em VM tem } \\
\text { risco aumentado de infecções respiratórias, } \\
\text { mas as medidas preventivas tiveram maior } \\
\text { impacto sobre elas. Mortalidade na UTI } \\
\text { pediátrica caiu de } 28,4 \text { para } 16,6 \% \text {, além } \\
\text { disso resultou na diminuição dos recursos de } \\
\text { saúde. }\end{array}$ \\
\hline
\end{tabular}




\begin{tabular}{|c|c|c|c|c|}
\hline A-17 & $\begin{array}{l}\text { The Effect of a Designed } \\
\text { Respiratory Care Program on the } \\
\text { Incidence of VentilatorAssociated } \\
\text { Pneumonia: A Clinical Trial }\end{array}$ & $\begin{array}{l}\text { Abbasinia, Bakhtiari, } \\
\text { Yazdannik, \& Babaii, } \\
2016 .\end{array}$ & II & $\begin{array}{l}\text { Os resultados deste estudo mostraram que } \\
\text { um programa de cuidados respiratórios } \\
\text { superiores reduziu a incidência de PAV de } \\
17,18 \% \text { para } 3,48 \% \text {. }\end{array}$ \\
\hline A-18 & $\begin{array}{l}\text { O impacto da aprendizage m } \\
\text { interprofissio nal na pneumonia } \\
\text { associada à ventilação: } \\
\text { implementaç ão de bundles em } \\
\text { uma unidade de cuidados } \\
\text { intensivos }\end{array}$ & $\begin{array}{l}\text { Coelho, Vieira, Leite, } \\
\quad \& \text { Lucas, } 2019\end{array}$ & III & $\begin{array}{l}\text { A observação direta dos profissionais } \\
\text { apontou que houve aumento significativo (p } \\
<0,05) \text { da HM de } 88,81 \% \text { para } 94,88 \% \text { após } \\
\text { a aspiração. A aspiração subglótica, embora } \\
\text { não significativa (p = 0,170), também } \\
\text { aumentou } 18,75 \% \text {, comparando os períodos } \\
\text { pré e pósintervenção. Essa taxa pode indicar } \\
\text { o conhecimento fragmentado da equipe } \\
\text { sobre as medidas de HM e aspiração } \\
\text { subglótica. }\end{array}$ \\
\hline A-19 & $\begin{array}{c}\text { Impactos e fatores determinante s } \\
\text { no bundle de pneumonia associada } \\
\text { à ventilação mecânica }\end{array}$ & $\begin{array}{l}\text { Rodrigues, Fragoso, } \\
\text { Beserra, \& Ramos, } \\
2016\end{array}$ & IV & $\begin{array}{l}\text { O estudo concluiu que a adoção de um } \\
\text { protocolo é complexa, pois muitas variáveis } \\
\text { atuam diretamente sobre o mesmo, como se } \\
\text { pode verificar. A implementação } \\
\text { inadequada por treinamento deficitário, a } \\
\text { falta de insumos necessários e supervisão } \\
\text { contínua para garantia da continuidade do } \\
\text { processo, levam a falhas relacionadas à } \\
\text { aplicação. }\end{array}$ \\
\hline
\end{tabular}

Fonte: Autores.

Os anos de 2016 e 2019 aparecem como os de maior produção, considerando-se que 10 artigos foram publicados nesse período. Evidenciou-se que os anos de 2015, 2017 e 2020 tiveram duas publicações cada um; no ano de 2018 foram evidenciadas três produções acerca da temática, somando 19 no total.

Dessa forma, foram definidas as seguintes categorias: Categoria I- Ações de prevenção para o enfrentamento da PAV e Categoria II- Estratégia de promoção para enfrentamento da PAV. Sendo 14 dos artigos determinados pelos parâmetros escolhidos se adequaram à categoria I. Seis dos artigos determinados pelos parâmetros escolhidos se adequaram à categoria II.

De acordo com análise feita, os cuidados Higienização oral e Elevação da Cabeceira foram os mais frequentes, presentes em seis artigos cada um. O cuidado Pressão-Cuff presente em cinco artigos; o cuidado Aspiração de secreções subglóticas em três artigos; o cuidado Desmame Precoce da sedação em três artigos e o cuidado higienização das mãos em três artigos.

\section{Discussão}

\subsection{Ações de prevenção para o enfrentamento da PAV}

PAV é uma IRAS e pode ser evitada através de medidas que vão garantir a segurança, manutenção da vida e um bom prognóstico para o paciente. A literatura possui estudos clínicos acerca dos assuntos que foram bastante proveitosos para esta pesquisa, contribuindo assim ao objetivo deste estudo a respeito das medidas preventivas de pneumonia associada à ventilação mecânica. Considerando a importância e a complexidade da PAV, faz-se necessária a realização de intervenções que causem impactos significativos à sua prevenção.

\subsubsection{Higiene oral com clorexidina}

Os artigos que abordaram o cuidado higienização oral foram A-2, A-8, A-12, A-13, A-18, estes relatam que houve boa adesão do cuidado pela equipe de profissionais de saúde, também mostraram resultados satisfatórios na diminuição da incidência de PAV. 
A limpeza da cavidade oral consiste em ações mecânicas (suç̧ão) e químicas (clorexidina 0,12\%), que fornecem um meio de prevenção contra PAV significativamente eficiente em paciente em uso de ventilação mecânica. Como a cavidade oral fornece meios satisfatórios para crescimento de patógenos causadores de PAV, um protocolo diário de higiene oral resulta em diminuição da mortalidade, consequentemente a diminuição do tempo e custos de internação (Scalco et al., 2019).

Desse modo, a higiene bucal deve ser realizada com swab e clorexidina $0,12 \%$, a cada 12 horas. Silva et al (2020) sugestiona que é importante a inclusão da equipe odontológica no atendimento ao paciente melhorando a contribuição da atenção à saúde bucal do paciente em VM, sendo assim, implementando um novo protocolo de higiene bucal com clorexidina $0,12 \%$ para prevenção de infecções associadas ao ambiente de UTI.

Lourençone, Branco, Monteiro, Fonseca, e Caregnato (2019) demonstra em seu estudo uma boa adesão dos profissionais a higienização oral com clorexidina. Após avaliação contínua executada 32 por um profissional, um protocolo direcionado revelou aumento para 84,9 \% na taxa de adesão para esta medida e como resultado diminuição significativa da PAV.

Diversos fatores colaboram para modificações no cuidado bucal, compreendendo capacitação e prática dos profissionais, além da disponibilidade de recursos. Porém o uso diário e correto da clorexidina duas vezes ao dia, evita a formação de biofilme e redução de patógenos gram-negativos, reduzindo assim o risco de PAV (Coelho, Vieira, Leite, \& Lucas, 2019).

Dessa maneira, sendo a higiene bucal um cuidado de relevância na prevenção da PAV, é de suma importância incentivar a participação e responsabilidade de todos os sujeitos envolvidos. Sendo assim, a divisão do cuidado deverá contribuir para efetividade no processo de cuidar (Landelle et al., 2018).

\subsubsection{Elevação da cabeceira}

A elevação da cabeceira em 30-45 foi abordada nos artigos A-5, A-6, A-8, A-9, A-17, A-18.

Trata-se de uma das ações mais simples e fáceis que podem reduzir a incidência de PAV. A necessidade dessa intervenção se dá pelo fato de que os pacientes não sofrem broncoaspiração e consequentemente não ocorre a PAV. Segundo a Associação Americana de Enfermagem em UTI a recomendação é elevar entre 30 e $45^{\circ}$ com os pacientes em VM. O resultado se torna eficiente se iniciar nas primeiras 24h, sendo uma intervenção recomendada (Abbasinia, Bakhtiari, Yazdannik, \& Babaii, 2016).

Segundo de Almeida et al., (2015), a elevação da cabeceira contribui também para uma melhoria da corrente ventilatória, vindo a diminuir a atelectasia. Enfatiza que é uma prática simples, com muitos benefícios e deve ser valorizada pelos profissionais, porém deve ser realizada com cuidados, responsabilidade e supervisionamento.

Com isso, evidencia-se que a cabeceira elevada acima de $30^{\circ}$, em relação à posição supina, favorece as trocas gasosas, diminuição do refluxo gastroesofágico e a colonização da orofaringe, evitando aspiração do conteúdo gástrico. A posição supina promove atelectasia e fluxo pulmonar venosos alterado aumentando as chances do paciente adquirir PAV, confirmando a efetividade da cabeceira acima de $30^{\circ}$ como um método eficaz na prevenção de PAV (Cruz, 2018).

Coelho et al.,(2019) apresentou em seu estudo adesão de 90, 38\% dos profissionais na realização da elevação da cabeceira entre $30^{\circ}$ e $45^{\circ}$, o objetivo dessa intervenção é evitar o mau posicionamento do paciente no leito, que se torna fator de risco para desenvolvimento de PAV. Além disso, após comparação da posição semi- inclinada com a posição supina, a primeira demonstrou redução significativa no risco de desenvolver a PAV em comparação com a segunda.

Apesar de ser uma medida preconizada pela Anvisa, de fácil execução e baixo custo, existe ainda empecilhos na sua realização que levam a uma baixa adesão dos profissionais. Dentre elas estão: manipulação da cabeceira por muitos profissionais durante o dia e instabilidade no quadro hemodinâmico do paciente (Lourençone et al., 2019).

Denota-se que é recomendado para o pacote de prevenção de PAV em adultos a elevação da cabeceira da cama de $30^{\circ}$ $45^{\circ}$ para reduzir o risco de aspiração de conteúdo orofaríngeo e gastrointestinal contaminado. Desse modo, preconiza que a 
posição semirreclinada reduz a taxa de PAV clinicamente suspeita e microbiológica confirmada. A princípio a elevação da cabeceira da cama é correta, sendo encontrada em quase todos os pacotes de redução de PAV e sua implementação é fácil e aceita pelos profissionais de saúde (Azab et al., 2015).

\subsubsection{Pressão do cuff}

O cuidado pressão-cuff do tubo endotraqueal foi apresentado pelos artigos A- 5, A-8, A-16, A-17, A-19, consiste na verificação do valor da pressão que deve estar entre 20 a $30 \mathrm{~cm} \mathrm{H2O}$, é de suma importância a permanência dos valores adequados, pois evita a broncoaspiração de secreções orofaríngeas para as vias aéreas inferiores, reduzindo assim os riscos de desenvolvimento da PAV.

A manutenção da pressão adequada do cuff tem como finalidade assegurar a vedação da traqueia para impedir microaspirações de secreções subglóticas para o trato respiratório inferior, impedindo assim que bactérias encontrem um lugar propício para proliferação, consequentemente impedindo a PAV (Rodrigues, Fragoso, Beserra, \& Ramos, 2016).

Cruz (2018) destacam em seu estudo que a pressão ideal do Cuff é acima de $20 \mathrm{cmH} 2 \mathrm{O}$, pois um valor inferior a esse está relacionado ao desenvolvimento de PAV. Porém, persiste uma dúvida acerca da permeabilização plena na medição, pois na remoção do medidor ocorre fuga, gerando uma incerteza da pressão exata do Cuff pelos profissionais.

Segundo Abbasinia et al., (2016) a medição deve ocorrer uma vez por turno, visto que o balão não insuflado de forma correta pode ocasionar necrose da traqueia ou fístula traqueoesofágica. Peña-lópez et al., (2016) complementa que os resultados positivos foram nos pacientes que tinha Cuff mantido acima de $20 \mathrm{cmH} 2 \mathrm{O}$ e que as chances de se adquirir PAV aumentam em 4 vezes se pressão mantida abaixo de $20 \mathrm{cmH} 2 \mathrm{O}$, cita ainda sobre os cuidados relativos ao vazamento do Cuff e consequentemente aspiração de secreções.

Foi observado no estudo de Lourençone et al., (2019) que a aderência a esta medida apresentou aumento geral durante seis meses de monitoramento. Refere em seu estudo uma boa taxa de adesão à pressão do Cuff, principalmente pela equipe de enfermagem, no geral com $91,7 \%$ de adequação. Sendo considerado feita inadequadamente, na maioria dos casos, aquelas que não foram realizadas o mínimo de vezes que constava no protocolo.

Destaca-se que a adesão a pressão do balonete aumentou durante o estudoe que foi citada por grande parte dos profissionais como medida importante na prevenção da PAV. Demonstrou que esse critério reduz significativamente a incidência de pneumonia em pacientes com ventilação mecânica invasiva (VMI) (Rodrigues et al., 2016).

\subsubsection{Higienização das mãos}

O cuidado de higienização das mãos foi mencionado nos artigos A-6, A-13 e A-18.

Destaca-se que a higienização das mãos (HM) é o método mais eficaz na prevenção de IRAS, porém infelizmente apresenta baixa adesão nos serviços de saúde. A HM apresenta valor significativo em relação ao tempo de trabalho, reforça que a quantidade de tempo de trabalho influencia em sua adesão. Ainda destaca que a frequência com que os profissionais realizem a HM nos momentos mais adequados para segurança do paciente aumentou após intervenção educacional (Coelho et al., 2019).

Landelle et al., (2018) avaliou a HM como uma das principais medidas desde o período pré-internação, aumentando significativamente ao longo do período de internação do paciente, sendo assim melhor aceita entre os profissionais de saúde, consequentemente aumentando o nível de segurança e diminuindo as chances do paciente adquirir qualquer IRAS.

Com isso, destaca-se que a HM é a mais importante intervenção de controle de infecção em todos os serviços de saúde, mas consequentemente é uma das estratégias mais difíceis de manter. Desse modo, campanhas de treinamento de HM devem ser realizadas, contendo as 6 etapas de lavagem das mãos são fundamentais para melhor o entendimento e a importância da higienização correta (Azab et al., 2015). 


\subsubsection{Aspiração de secreção subglótica}

Os artigos que ressaltaram o cuidado de aspiração subglótica foram A-5, A-17, A-18.

A aspiração de secreções subglóticas é um procedimento que permite manter as vias aéreas abertas e devidamente ventiladas, no qual deve ser realizado de forma asséptica, por uma sonda conectada a um sistema de vácuo, introduzida no tubo orotraqueal ou traqueostomia. Sendo assim, as normas preconizam que o doente deve estar em decúbito dorsal com a cabeceira da cama ligeiramente elevada (30- 45 ) para evitar o elevado risco de broncoaspiração (Cruz, 2019).

Esse procedimento é indispensável na prevenção da PAV, Coelho et al., (2019) teve em seu estudo adesão aumentada após a intervenção. Um outro ponto mostra que a aspiração de secreção subglótica reduziu a incidência de PAV, dias em VM e custos antimicrobianos em pacientes submetidos à cirurgia cardíaca.

Abbasinia et al., (2016) demonstrou em seu estudo que há aspiração em pacientes ventilados mecanicamente a cada duas horas independente da mudança de decúbito do paciente reduz significativamente a PAV.

\subsubsection{Desmame precoce da sedação}

O cuidado desmame precoce da sedação foi mencionado pelos artigos A-9, A13, A-18, A-19.

Os pacientes com VM recebem infusão contínua de sedação para aliviar o desconforto e a dor. Logo, tornam-se suscetíveis ao risco de sedação prolongada e, consequentemente suscetíveis à PAV devido e redução do reflexo da tosse resultando no alto risco de aspiração. Essa medida reduz os dias passados no ventilador, e consequentemente as infecções que estão relacionadas ao uso prolongado da VM (Coelho et al., 2019).

Uma recomendação importante é a interrupção diária da sedação, pois é possível avaliar o padrão respiratório do paciente (considerando que pacientes acordados podem contribuir no desmame ventilatório), assim como a possibilidade de extubação dos pacientes, isso para reduzir tempo de VM, e por consequência a contribuição para prevenir PAV. Neste contexto, cabe à equipe multidisciplinar discutir quando será suspensa a sedação e ficar atenta às reações do paciente, devendo ainda monitorá-lo para evitar a extubação acidental e quedas da cama ou maca (de Almeida et al., 2015).

Desse modo, a avaliação do nível de sedação é feita porque quanto maior o nível de sedação mais dificultoso o desmame ventilatório e pode culminar em maior risco para PAV. A interrupção diária da sedação está associada a maior sobrevida dos pacientes submetidos à ventilação mecânica (Rodrigues et al., 2016).

Enfatiza-se que a tentativa diária de desmame da VM se mostra eficaz para prevenção de PAV, pois diminui tempo da VM, diminuindo as taxas dos riscos para adquirir PAV, sendo assim uma medida eficaz para uma boa adesão entre a equipe multiprofissional, sendo totalmente indicada (Landelle et al., 2018).

\subsection{Estratégia de promoção para enfrentamento da PAV}

Os maiores e melhores investimentos são feitos na prevenção. Quando se trata de PAV, pode ser ressaltado as medidas preventivas, porém não pode ter medidas caso não tenha uma equipe qualificada para aplicar essas medidas na prática. Logo, faz-se necessário a criação de um programa educacional de forma continuada, para esclarecer e treinar a equipe sobre os conceitos de segurança do paciente e fornecer informações de como implementar as medidas de prevenção de PAV. É comprovado que a educação permanente é uma das maiores qualificações em prevenção, sobretudo quando há mudanças nos protocolos, deve-se ofertar o treinamento adequado, garantindo a sistematização das intervenções oferecidas (Coelho et al., 2019).

A educação profissional, é uma grande aliada para o sucesso da implantação de um bundle preventivo, conhecimento e conscientização da equipe. Todos os profissionais de enfermagem devem fortalecer a necessidade e a importância da aplicação das medidas de prevenção e seu impacto na redução dos indicadores de PAV em UTI. Com isso, para que a educação realmente tenha impacto, é necessária a supervisão contínua do bundle para uma implementação adequada. Entretanto, preparar um 
feedback sobre o comportamento e a influência dos valores da PAV é de suma importância para que os profissionais se sintam como parte do processo de mudanças, responsáveis pelo sucesso dos indicadores e pela promoção de um cuidado seguro (Branco et al., 2020).

Todavia, a competência profissional limitada impede que os profissionais empreguem as medidas eficazes para prevenção da PAV. Destaca-se que o conhecimento acerca dos protocolos e de como realizá-los é um pré-requisito importante para prevenção da PAV. O conhecimento limitado impede a compreensão e leva a uma gestão ineficaz dessas medidas. Além disso, existe uma baixa motivação dos profissionais que afeta a capacidade de implementar medidas preventivas da PAV, dentre os fatores estão: condições de trabalho desfavoráveis, carga de trabalho pesada, déficit na escala dos profissionais, extensas jornadas de trabalho (Atashi, Yousefi, Mahjobipoor, \& Yazdannik, 2018).

Ressaltou-se que $42 \%$ dos profissionais relatou não ter recebido educação sobre PAV, sendo a falta de educação e treinamento dos profissionais um fator contribuinte para alta taxa PAV. Destaca-se que houve significativa melhora na comunicação, aplicação e diminuição da incidência de PAV após educação dos profissionais relacionada ao tema (Aloush, 2018).

Outro fator que pode ser somado aos anteriores, são as atitudes profissionais que afetam a prática da prevenção de PAV. Notou-se que eles não realizavam as medidas, pois não acreditavam em sua ação benéfica. Ressalta-se a importância da implementação de capacitações que reforcem junto aos profissionais a relevância da utilização das medidas preventivas na assistência ao paciente em uso de VM, pois são consideradas vitais e de grande relevância para sucesso na prestação de cuidados a vida (Atashi et al., 2018).

De Barros (2019) apontam que a implementação de pacotes de medidas a prevenção de PAV um método de sucesso na prática clínica. Mas para ter sucesso a equipe multiprofissional deve eleger as intervenções conforme a necessidade de cada unidade. Entretanto, um dos grandes desafios na atualidade é que a conformidade das intervenções elencadas nos bundles tenha uma satisfatória adesão, em longo prazo, mantendo uma implementação conjunta a outras medidas de prevenção de PAV. A execução correta de todos os cuidados do pacote de medidas é eficaz na redução de infecções, com isso, a implementação pacote de bundles resulta em uma redução de PAV.

Yilmaz et al., (2016) reforça que o controle de infecções hospitalares só é possível se conjugado com medidas preventivas e monitoramento dos níveis de infecções, identificando e corrigindo os problemas. Os métodos de treinamento geralmente usados que apresentam maiores resultados incluem palestras, apresentações de vídeos, pôsteres, pesquisas e sessões de prática. Bons níveis de treinamento foram alcançados através dos testes de avaliação de conhecimento para garantia da sua qualidade. Enfatiza ainda que houve aumento de 26\% em relação a HM e uma diminuição de 31,7\% nos níveis de PAV após o treinamento. Conscientizando a equipe de seus deveres, a capacitação se torna indispensável para prevenir e minimizar possíveis infecções.

\section{Consideracões Finais}

A PAV é uma das IRAS que tem uma maior incidência e prevalência elevada nas Unidades de Terapia Intensiva em diversos hospitais. Dentre os seus agravos está a piora do prognóstico do paciente, sendo ainda maiores as chances de complicações e tempo de permanência em UTI's, aumentando também os custos com tratamentos, sendo evidente que a adoção de medidas preventivas apresenta um impacto positivo na diminuição da ocorrência desta infecção em unidades críticas.

Assim, é de suma importância a notificação dos casos de PAV para maior asseguramento e uma diminuição ao longo do tempo com treinamentos e ações educativas multidisciplinares. A aplicação do protocolo tem como finalidade de implementar, planejar e sensibilizar os profissionais, garantindo os materiais para a execução das medidas preconizadas pelo bundle, a fim de garantir uma qualidade assistencial e segurança ao paciente submetido à VM.

Com isso, a implementação de práticas baseadas em evidências, mostraram ser importantes ferramentas que devem ser 
incorporadas diariamente na assistência, mas não são uma tecnologia muito conhecida sendo pouco aplicada pelas equipes. Desse modo, após aplicação do protocolo foi observado baixa taxa de infecção nos pacientes que receberam o pacote. Além disso, compreende-se que é necessária uma educação em saúde aos profissionais que atuam nas UTI's, para que haja uma melhor adesão das medidas preventivas.

Ressalta-se que o aperfeiçoamento do profissional de saúde a respeito do pacote de bundle e a educação em saúde é de suma importância e tem um papel importante no serviço prestado ao paciente internado em uma UTI, reduzindo o risco de PAV que se torna uma complicação decorrente ao paciente hospitalizado.

Entretanto, são necessárias mais pesquisas acerca das práticas de prevenção da PAV, para esclarecer questionamentos, ajudar na tomada de decisões e apoiar na implementação dessas medidas, que repercutirão na qualidade da assistência aos pacientes em uso de VM.

Deixa- se como sugestão a expansão de estudos a cerca dessa temática, com o intuito de esclarecer e enfatizar as intervenções mais eficazes a serem incorporadas em protocolos hospitalares, além disso favorecer a adesão as medidas de prevenção a PAV.

\section{Referências}

Abbasinia, M., Bahrami, N., Bakhtiari, S., Yazdannik, A., \& Babaii, A. (2016). The effect of a designed respiratory care program on the incidence of ventilatorassociated pneumonia: a clinical trial. Journal of caring sciences, 5(2), 161.

Agência Nacional de Vigilância em Saúde (2015). Programa Nacional de Prevenção e Controle de Infecções Relacionadas à Assistência em Saúde(2013-2015). http://portal.anvisa.gov.br/documents/33852/272166/Programa+Nacional+de+Preven\%C3\%A7\%C3\%A3o+e+Controle+de+Infec\%C3\%A7\%C3\%B5es+Relac ionadas+\%C3\%A0+Assist\%C3\%AAncia+\%C3\%A0+Sa\%C3\%BAde+\%2820132015\%29/d1d0601f-004c-40e7-aaa5-0af7b32ac22a

Alecrim, R. X., Taminato, M., Belasco, A., Longo, M. C. B., Kusahara, D. M., \& Fram, D. (2019). Estrategias para la prevención de la neumonía asociada a la ventilación mecánica: revisión integrativa. Revista Brasileira de Enfermagem, 72(2), 521-530.

Aloush, S. M. (2018). Nurses' implementation of ventilator-associated pneumonia prevention guidelines: an observational study in Jordan. Nursing in critical care, 23(3), 147-151.

Araújo, B. T., \& Pereira, D. C. R. (2017). Políticas para controle de Infecções Relacionadas à Assistência à Saúde (IRAS) no Brasil, 2017.

Atashi, V., Yousefi, H., Mahjobipoor, H., \& Yazdannik, A. (2018). The barriers to the prevention of ventilator-associated pneumonia from the perspective of critical care nurses: A qualitative descriptive study. Journal of clinical nursing, 27(5-6), e1161-e1170.

Azab, S. F., Sherbiny, H. S., Saleh, S. H., Elsaeed, W. F., Elshafiey, M. M., Siam, A. G., \& Gheith, T. (2015). Reducing ventilator-associated pneumonia in neonatal intensive care unit using "VAP prevention Bundle": a cohort study. BMC infectious diseases, 15(1), 1-7.

Botelho, L. L. R., de Almeida Cunha, C. C., \& Macedo, M. (2011). O método da revisão integrativa nos estudos organizacionais. Gestão e sociedade, 5(11), $121-136$.

Branco, A., Lourençone, E. M. S., Monteiro, A. B., Fonseca, J. P., Blatt, C. R., \& Caregnato, R. C. A. (2020). Education to prevent ventilator-associated pneumonia in intensive care unit. Revista Brasileira de Enfermagem, 73(6).

Coelho, A. P., Vieira, R. A. M., Leite, M. A., \& Lucas, T. C. (2019). O impacto da aprendizagem interprofissional na pneumonia associada à ventilação: implementação de bundles em uma unidade de cuidados intensivos. Enferm. foco (Brasília), 93-100.

Cruz, J. R. M. D. (2018). Pneumonia associada à ventilação mecânica invasiva: cuidados de enfermagem (Doctoral dissertation).

De Almeida, K. M. V., de Barros, O. M. C., Santos, G. J. C., Valença, M. P., de Almeida Cavalcanti, A. T., \& Ferreira, K. O. (2015). Adesão às medidas de prevenção para pneumonia associada à ventilação mecânica. Revista de Enfermagem da UFSM, 5(2), 247-256.

De Barros, F. R. B. (2019). Adhesión al bundle de prevención de neumonía asociada a la ventilación mecánica. Revista Cuidarte, 10(2).

Dutra, L. A., Esteves, L. D. O., Silva, T. O. D., Resck, Z. M. R., Lima, R. S., \& Sanches, R. S. (2019). Pneumonia associada à ventilação mecânica: percepção dos profissionais de enfermagem. Rev. enferm. UFPE on line, 884-892.

Ercole, F. F., Melo, L. S. D., \& Alcoforado, C. L. G. C. (2014). Revisão integrativa versus revisão sistemática. Revista Mineira de Enfermagem, $18(1)$, 9-12.

Freire, M. E. M., Sawada, N. O., de França, I. S. X., da Costa, S. F. G., \& Oliveira, C. D. B. (2014). Qualidade de vida relacionada à saúde de pacientes com câncer avançado: uma revisão integrativa. Revista da Escola de Enfermagem da USP, 48(2), 357-367.

Frota, M. L., Campanharo, C. R. V., Lopes, M. C. B. T., Piacezzi, L. H. V., Okuno, M. F. P., \& Batista, R. E. A. (2019). Good practices for preventing ventilatorassociated pneumonia in the emergency department. Revista da Escola de Enfermagem da USP, 53 
Research, Society and Development, v. 10, n. 5, e51010515207, 2021

(CC BY 4.0) | ISSN 2525-3409 | DOI: http://dx.doi.org/10.33448/rsd-v10i5.15207

Lagier, D., Platon, L., Lambert, J., Chow-Chine, L., Sannini, A., Bisbal, M., \& Mokart, D. (2017). Protective effect of early low-dose hydrocortisone on ventilator-associated pneumonia in the cancer patients: a propensity score analysis. Annals of intensive care, 7(1), 1-9.

Landelle, C., Boyer, V. N., Abbas, M., Genevois, E., Abidi, N., Naimo, S., \& Pugin, J. (2018). Impact of a multifaceted prevention program on ventilatorassociated pneumonia including selective oropharyngeal decontamination. Intensive care medicine, 44(11), 1777-1786.

Lourençone, A. M. S., Branco, A., Monteiro, A. B., Fonseca, J. P., \& Caregnato, R. C. A. (2019). Adesão às medidas preventivas versus incidência de pneumonia associada à ventilação mecânica. Rev. Epidemiol. Controle Infecç. Santa Cruz do Sul, 9(2), 142-148.

Maran, E., Spigolon, D. N., de Melo, W. A., da Silva Barreto, M., do Prado Tostes, M. F., \& Teston, E. F. (2019). Prevenção da pneumonia associada à ventilação mecânica sob a ótica de acadêmicos de enfermagem. Revista de Pesquisa: Cuidado é Fundamental, 118-123.

Melnyk, B. M., \& Fineout-Overholt, E. (Eds.). (2011). Evidence-based practice in nursing \& healthcare: A guide to best practice. Lippincott Williams \& Wilkins.

Peña-López, Y., Pujol, M., Campins, M., González-Antelo, A., Rodrigo, J. Á., Balcells, J., \& Rello, J. (2016). Implementing a care bundle approach reduces ventilator-associated pneumonia and delays ventilator-associated tracheobronchitis in children: differences according to endotracheal or tracheostomy devices. International Journal of Infectious Diseases, 52, 43-48.

Rau, C.; Santos, I. M. de A (2013). Pneumonia associada à ventilação mecânica: importância dos métodos preventivos em pacientes críticos. http://www.cpgls.pucgoias.edu.br/8mostra/Artigos/SAUDE\%20E\%20BIOLOGICAS/PNEUMONIA\%20ASSOCIADA\%20\%C3\%80\%20VENTILA\%C3\% 87 $\% \mathrm{C} 3 \% 83 \mathrm{O} \% 20 \mathrm{MEC} \% \mathrm{C} 3 \% 82 \mathrm{NICA} \% 20 \mathrm{IMPORT} \% \mathrm{C} 3 \% 82 \mathrm{NCIA} \% 20 \mathrm{DOS} \% 20 \mathrm{M} \% \mathrm{C} 3 \% 89 \mathrm{TODOS} \% 20 \mathrm{PREVENTIVOS} \% 20 \mathrm{EM} \% 20 \mathrm{PACIENTES} \% 20 \mathrm{CR} \%$ C3\%8DTICOS.pdf

Rodrigues, A. N., Fragoso, L. V., Beserra, F. D. M., \& Ramos, I. C. (2016). Impactos e fatores determinantes no bundle de pneumonia associada à ventilação mecânica. Revista Brasileira de Enfermagem, 69(6), 1108-1114.

Samra, S. R., Sherif, D. M., \& Elokda, S. A. (2017). Impact of VAP bundle adherence among ventilated critically ill patients and its effectiveness in adult ICU. Egyptian Journal of Chest Diseases and Tuberculosis, 66(1), 81-86.

Santos, C. R. D., Costa, B. H. S., Dias, T. K. C., Guedes, H. C. D. S., Paz, M. S. S. D., Oliveira, T. C. D., \& Marinho, H. L. M. (2018). Fatores de risco que favorecem a pneumonia associada à ventilação mecânica. Rev. enferm. UFPE on line, 3401-3415.

Scalco, J. D. M., Fernandes, T. M. F., Scalco, V. F., Ribeiro, E., Shiguematsu, S. M., \& Poleti, M. L. (2019). Analysis of the Preventative Influence of an Oral Hygiene Protocol on Ventilator-Associated Pneumonia. J. health sci.(Londrina), https-seer.

Silva, D. H. F., Camargos, J. H. D., Rodrigues, J. G., Nogueira, L. S., Azevedo, D. A. D., Carvalho, M. D. G., \& Pinheiro, M. D. B. (2020). Impact of oral hygiene in patients undergoing mechanical ventilation in the COVID-19 pandemic. Revista da Associação Médica Brasileira, 66, 96-101.

Su, K. C., Kou, Y. R., Lin, F. C., Wu, C. H., Feng, J. Y., Huang, S. F., \& Chang, S. C. (2017). A simplified prevention bundle with dual hand hygiene audit reduces early-onset ventilator-associated pneumonia in cardiovascular surgery units: An interrupted time-series analysis. PloS one, 12(8), e0182252.

Yilmaz, G., Aydin, H., Aydin, M., Saylan, S., Ulusoy, H., \& Koksal, I. (2016). Staff education aimed at reducing ventilator-associated pneumonia. Journal of medical microbiology, 65(12), 1378-1384. 